Conclusions Hypoxic preconditioning reverses the morbidity and mortality associated with acute hypoxia following intrapulmonary bacterial challenge. This response is dependent on the preconditioning of the innate immune system by suppressing HIF1 alpha and altering circulating leukocyte metabolism.

Support RSD is funded by the MRC. SRW is funded by the Wellcome Trust.

\section{S105 PNEUMOCOCCAL SEROTYPES IMPLICATED IN ADULT PNEUMOCOCCAL PNEUMONIA, 9 YEARS FOLLOWING THE INTRODUCTION OF THE INFANT VACCINE PROGRAMME IN THE UK}

${ }^{1} \mathrm{P}$ Daniel, ${ }^{1} \mathrm{D}$ Ashton, ${ }^{2} \mathrm{C}$ Sheppard, ${ }^{2} \mathrm{~S}$ Eletu, ${ }^{2} \mathrm{P}$ Sandu, ${ }^{2} \mathrm{D}$ Litt, ${ }^{2} \mathrm{~N}$ Fry, ${ }^{1} \mathrm{~W} S$ Lim. ${ }^{1}$ Department of Respiratory Medicine, Nottingham University Hospitals, Nottingham, UKi ${ }^{2}$ Respiratory and Vaccine Preventable Bacterial Reference Unit, London, UK

\subsection{6/thoraxjnl-2016-209333.111}

Background The introduction of the pneumococcal conjugate vaccines into infant vaccination schedules, has led to a change in the serotype prevalence causing adult pneumococcal disease, through the process of herd immunity. Whilst there are national surveillance programmes informing the changes in serotype in invasive pneumococcal disease, there are no comparable data to demonstrate the ongoing vaccine effect on non-invasive pneumococcal community acquired pneumonia (CAP), the most common clinical manifestation of pneumococcal disease in adults.

Methods Consecutive adult patients admitted to 2 hospitals, covering the catchment area of a large UK city, with a diagnosis of CAP were studied prospectively, over a 1 year period between September 2014 and 2015. A novel multiplex assay capable of detecting 24 serotypes/serogroups of Streptococcus pneumoniae was performed on patient urine. Pneumococcal infection was determined by identification of the organism from either sterile sites and/or detection of pneumococcal antigen or serotype in urine samples.

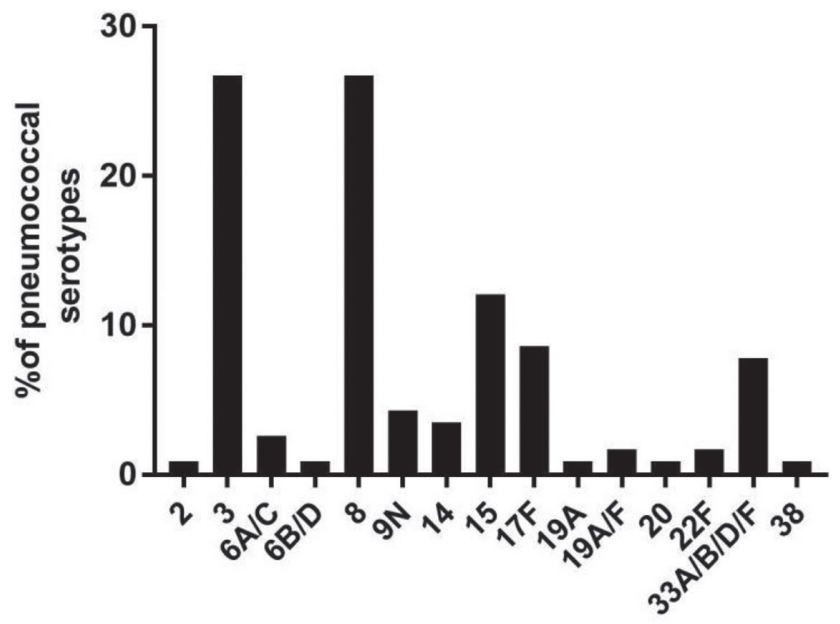

\section{Serotype}

\section{Abstract S105 Figure 1 Serotypes isolated in adult pneumococcal} CAP

Results Of 478 individuals admitted with CAP, pneumococcal disease was diagnosed in $166(34.7 \%)$ cases. Pneumococcal CAP diagnosis was made by blood culture, pneumococcal urinary antigen detection and urinary serotype detection in 23 (13.9\%), 61
(36.8\%) and 149 (89.8\%) cases respectively. A definitive single serotype was identified in 116 individuals; the most commonly observed were serotypes 3 and 8 (31 cases each, 26.7\%), followed by serogroup 15 (14 cases, 12.1\%), 17F (10 cases, 8.6\%) and $33 \mathrm{~A} / \mathrm{B} / \mathrm{D} / \mathrm{E}$ (9 cases, $7.8 \%$ ).

Conclusion This is the first report on extended serotype distribution implicated in adult pneumococcal CAP, 9 years after the introduction of the UK infant vaccination programme. In this era of high infant vaccine coverage, whilst the majority of isolates are non-vaccine types due to the effects of serotype replacement, serotype 3 remains a common cause of adult pneumococcal CAP and may reflect inadequate serotype specific vaccine effectiveness.

\section{S106 PERIPHERAL BLOOD NEUTROPHILS ARE PRIMED AND ACTIVATED IN BRONCHIECTASIS AND ARE ATTENUATED BY THE PRO-RESOLVING MEDIATOR LIPOXIN A4}

P Bedi, B McHugh, DJ Davidson, AG Rossi, AT Hill. MRC Centre for Inflammation Research, Edinburgh, UK

\subsection{6/thoraxjnl-2016-209333.112}

Introduction Excessive neutrophilic airways inflammation is the central feature of bronchiectasis but little is known about the role of serum neutrophils in bronchiectasis. Lipid mediators derived from arachidonic acid such as Lipoxin (LX)A4 are known to regulate the inflammatory process and generate pro-inflammatory, anti-inflammatory and pro-resolving mediators. In this research work, we propose to describe the function of peripheral neutrophils in bronchiectasis and the effect of LXA4.

Methods Three study groups were included in this study when clinically stable: 6 healthy volunteers; 6 patients with mild bronchiectasis with a Bronchiectasis Severity Index (BSI) score 0-4; 6 with severe bronchiectasis (BSI scores >9). Freshly isolated peripheral neutrophils from the groups were treated with LXA4 or vehicle control and we assessed spontaneous neutrophil apoptosis at 20 hours, neutrophil activation, neutrophil degranulation, phagocytosis of GFP labelled Pseudomonas aeruginosa and expression of LXA4 receptor formyl peptide receptor (FPR)2.

Results In vehicle treated neutrophils, there was increased viability and less apoptosis in bronchiectasis patients compared to healthy volunteers; Figure 1. There was a significant increase in CD11b upregulation; $\mathrm{p}=0.01$ and CD62L shedding; $\mathrm{p}=0.01$ in bronchiectasis patients compared to healthy volunteers. There was a significant increase in neutrophil degranulation with myeloperoxidase (MPO) release, in bronchiectasis patients; $\mathrm{p}=0.04$. There was an increase in neutrophil phagocytosis of GFP labelled Pseudomonas aeruginosa by neutrophils from bronchiectasis patients, $\mathrm{p}=0.03$, compared to healthy volunteers; Figure1.

In LXA4 treated neutrophils, there was no effect of LXA4 on spontaneous neutrophil apoptosis. There was a significant reduction in $\mathrm{n}$-formyl-methyl-leucyl-phenylalanine (fMLF)-induced CD11b upregulation and CD62L shedding by LXA4 in a dose dependent manner in all three groups. There was a significant reduction in cytochalasin-B and PMLF-induced activation of neutrophils and release of MPO, by LXA4 in all three groups. There was significant improvement in neutrophil phagocytosis of GFP labelled Pseudomonas aeruginosa in a dose dependent manner in all three groups. There was a statistically significant increase in FPR2 receptor expression in healthy volunteers compared to bronchiectasis patients when treated with LXA4 100nM, $\mathrm{p}=0.03$. 

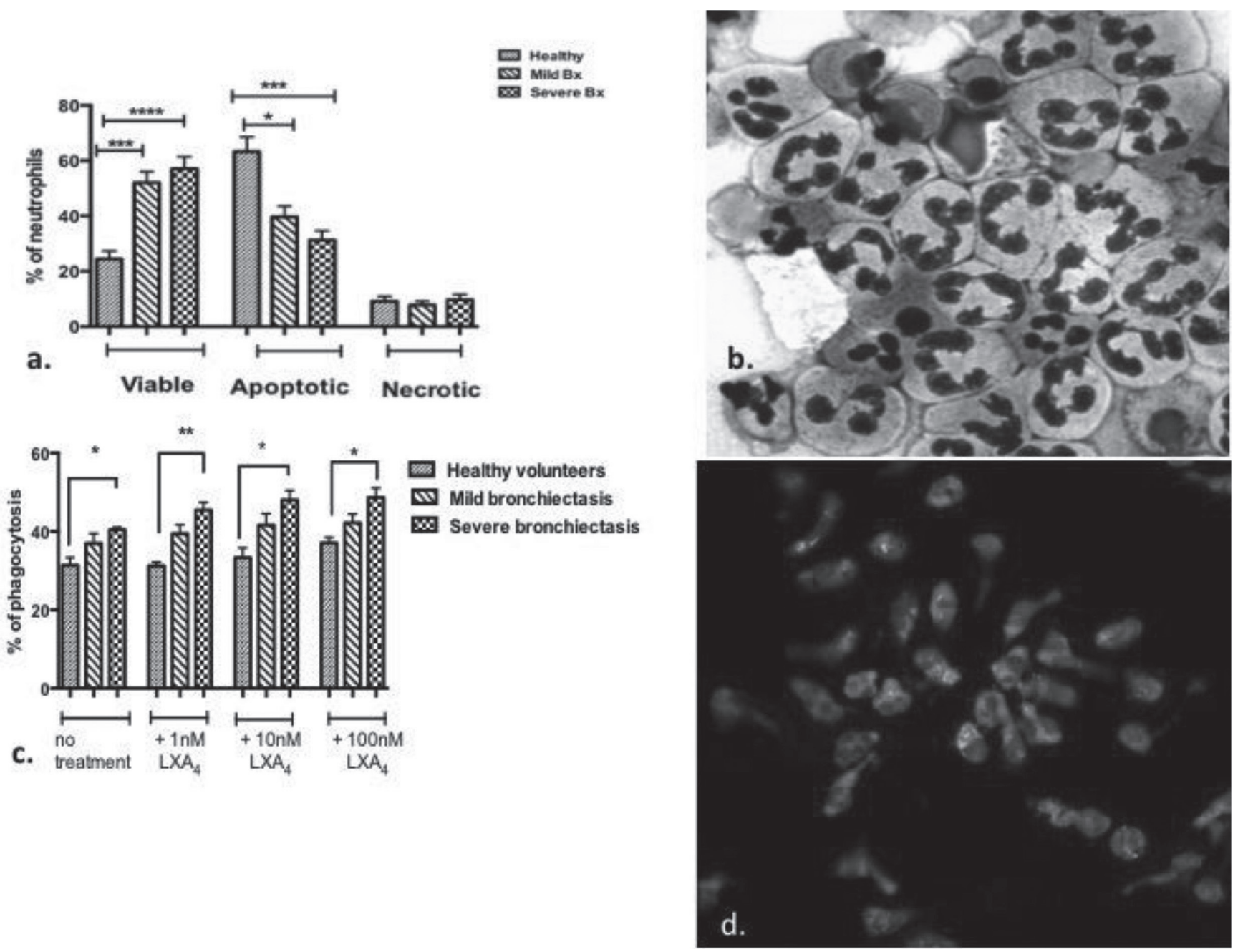

Abstract S106 Figure 1 a. Statistically significant increase in the \% of viable neutrophils and a decrease in apoptotic neutrophils in bronchiectasis; ${ }^{*} p=0.02,{ }^{* *} p=0.0003,{ }^{* * *} p<0.001$. b. Cytospin from a bronchiectasis patient after 20 hours apoptosis showing large numbers of viable neutrophils. c. Statistically significant increase in the \% of phagocytosis in bronchiectasis patients at baseline and increase with Lipoxin in a dose dependent manner; ${ }^{*} p=0.02,{ }^{* *} p=0.004$. d. Confocal image of phagocytosed GFP labelled Pseudomonas by neutrophil.

Conclusion Serum neutrophils in bronchiectasis are primed and activated compared to healthy volunteers. The pro-resolving mediator LXA4 stabilised the neutrophil whilst promoting neutrophil phagocytosis.

\section{Pulmonary Hypertension}

\section{S107 GENOTYPE-PHENOTYPE ASSOCIATIONS IN PULMONARY ARTERIAL HYPERTENSION CAUSED BY BMPR2 AND EIF2AK4 VARIANTS}

${ }^{1} \mathrm{C}$ Hadinnapola, ${ }^{1} \mathrm{M}$ Haimel, ${ }^{1} \mathrm{M}$ Bleda, ${ }^{2} \mathrm{H}$ Bogaard, ${ }^{3} \mathrm{G}$ Coghlan, ${ }^{4} \mathrm{P}$ Corris, ${ }^{5} \mathrm{~S}$ Gibbs, ${ }^{6} \mathrm{D}$ Kiely, ${ }^{7} \mathrm{~A}$ Lawrie, ${ }^{8} \mathrm{~A}$ Peacock, ${ }^{9} \mathrm{~J}$ Pepke-Zaba, ${ }^{10} \mathrm{~L}$ Southgate, ${ }^{9} \mathrm{M}$ Toshner, ${ }^{10} \mathrm{R}$ Trembath, ${ }^{2} \mathrm{~A}$ Vonk Noordegraaf, ${ }^{11} \mathrm{~J}$ Wharton, ${ }^{11} \mathrm{M}$ Wilkins, ${ }^{12} \mathrm{SJ}$ Wort, ${ }^{1} \mathrm{~S}$ Graf, ${ }^{1} \mathrm{NM}$ Morrell. ${ }^{1}$ Cambridge University, Cambridge, UK; ${ }^{2}$ VU Medical Centre, Amsterdam, Netherlands; ${ }^{3}$ Royal Free Hospital, London, UK; ${ }^{4}$ Newcastle University, Newcastle, UK; ${ }^{5}$ Hammersmith Hospital, London, UK; ${ }^{6}$ Royal Hallamshire Hospital, Sheffield, UK; ${ }^{7}$ Sheffield University, Sheffield, UK; ${ }^{8}$ Golden Jubilee Hospital, Glasgow, UK; ${ }^{9}$ Papworth Hospital, Cambridge, UK ${ }^{10}$ Kings College, London, UK; ${ }^{11}$ Imperial College, London, UK; ${ }^{12}$ Royal Brompton Hospital, London, UK

10.1136/thoraxjnl-2016-209333.113
Introduction Idiopathic pulmonary arterial hypertension (IPAH) is a rare and incurable disease. Causal mutations in BMPR2 are found in $17 \%$ of patients. Pulmonary veno-occlusive disease (PVOD) and pulmonary capillary haemangiomatosis $(\mathrm{PCH})$ are rarer forms of pulmonary hypertension and have a worse prognosis. Biallelic mutations in EIF2AK4 have been described in PVOD and PCH. We hypothesised that mutations in these genes are associated with specific phenotypes or endotypes.

Methods Whole genome sequencing was performed on genomic DNA from PAH patients recruited to the NIHR BRIDGE Study $(\mathrm{n}=679)$. Rare (absent from BRIDGE control cohorts $[\mathrm{n}=5906]$ and minor allele frequency $<0.0001$ in the ExAC database [http://exac.broadinstitute.org]) and predicted deleterious (CADD score $>15$ and Polyphen not benign) variants were selected for association testing with phenotypic and metabolomic data. Plasma samples from 288 patients were sent to Metabolon (USA) for a high-throughput metabolomic screen.

Results Mutations in BMPR2 (82 single nucleotide variants and 13 deletions) were identified in $14 \%$ of PAH patients. Unexpectedly, 22 rare and predicted deleterious EIF2AK4 variants were found in 17 patients with IPAH. Biallelic EIF2AK4 variants were found in $1 \%$ of patients (5 homozygous variant carriers and 4 potential compound heterozygotes). Additionally, there were 8 